\title{
The Combination of Respiratory Comorbidity and Neoadjuvant Chemoradiotherapy May Double the Risk of Anastomotic Leaks After Esophagectomy: Do We Know Enough to Tailor Neoadjuvant Therapies, or Take Other Preemptive Measures in High-Risk Patients?
}

\author{
Magnus Nilsson, $\mathrm{MD}, \mathbf{P h D}^{1,2}$ \\ ${ }^{1}$ Division of Surgery, Department of Clinical Science, Intervention and Technology (CLINTEC), Karolinska Institutet, \\ Stockholm, Sweden; ${ }^{2}$ Department of Upper Abdominal Diseases, Karolinska University Hospital, Stockholm, Sweden
}

The article by Klevebro et al., "Cardiorespiratory comorbidity and postoperative complications following esophagectomy: A European multicenter cohort study,"1 published in this issue of Annals of Surgical Oncology, provides new and important insights regarding the impact of comorbidity on complications after esophagectomy, and intriguingly also suggests that there is an interaction between respiratory complications and the type of neoadjuvant therapy. In fact, this study shows a significantly increased risk of anastomotic leaks and pulmonary complications in patients with respiratory comorbidity after neoadjuvant chemoradiotherapy, but not after neoadjuvant chemotherapy or in patients who went straight to surgery.

Another interesting finding is that patients with left ventricular ejection fraction (LVEF) $>55 \%$ had only onethird as many anastomotic leaks as those with LVEF < $55 \%$. Unfortunately, the sample size of patients with LVEF data available was too small to permit analyses stratified by type of neoadjuvant therapy. In addition, this study replicates the already well-established findings that American Society of Anesthesiologists (ASA) score and performance status can predict postoperative morbidity after esophagectomy, and that age, when adjustment is made for its frequent companion comorbidity, does not seem to strongly predict postoperative complications.

\section{(C) Society of Surgical Oncology 2019}

First Received: 15 May 2019;

Published Online: 21 June 2019

M. Nilsson, MD, PhD

e-mail: magnus.nilsson@ki.se
So, what do we do with this information? Of course, the novel findings of this study need to be replicated in new well-designed studies, for example as suggested by the authors themselves in the two ongoing large randomized phase III trials Neo-AEGIS ${ }^{2}$ and ESOPEC, ${ }^{3}$ comparing perioperative chemotherapy with neoadjuvant chemoradiotherapy. As data from these trials will not be available for many years, a good short-term option would be to see if the findings can be repeated in national registry cohorts, for example those from The Netherlands or the Scandinavian countries.

If reasonable confirmation is achieved, the most obvious adaptation of practice would be severe caution with neoadjuvant chemoradiotherapy in patients with known respiratory comorbidity or with lung function testing, especially a FEV1/FVC quota of $<70 \%$, indicating preoperative respiratory impairment. There are to date no robust data showing a survival benefit for neoadjuvant chemoradiotherapy compared with neoadjuvant chemotherapy in patients in general. Perioperative chemotherapy or surgery alone might be more appropriate treatment options for patients with cardiorespiratory comorbidity.

A difficult issue regards where to draw the line for operability as a function of surgical risk, in patients with severe cardiopulmonary comorbidity. Future research needs to determine which cardiopulmonary function tests to use, and what cutoff levels to apply for treatment modification on an individual level. An increased risk of anastomotic complications is in most cases not by far reason enough to abstain from surgery, the best curative therapy option available, as the ability to treat and salvage 
in severe complication situations is very high today. In this perspective, we need to seek the right denominator. Being very selective in choosing only fit patients for curative surgery produces nice surgical results with few leaks, low overall morbidity, and close to no mortality, but only cures a very small proportion of the patients diagnosed with esophageal or junctional cancers. Accepting also higherrisk patients for surgery will result in less impressive surgical results but may cure and prolong life with reasonable quality of life in a considerably larger proportion of the patients. However, there is of course a reasonable limit, and defining this limit is one of the most important tasks of research in the near future.

Finally, I would like to make a bold suggestion. Despite the high salvage capacity in high-volume esophageal surgery centers, an anastomotic leak in a patient with severe cardiopulmonary comorbidity entails a high risk of death and great suffering for the patient and relatives, and drives high costs. With the current study's findings of greatly increased risks of anastomotic leaks in patients with respiratory comorbidity and with left ventricular ejection fraction $<55 \%$, it would be possible to accurately identify high-risk patients for leak. An option could then be to use preemptive, intraluminal Eso-SPONGE vacuum therapy for 5-6 days in all high-risk patients. This was suggested and tested in a small single-center phase I trial from Zürich, in which 19 anastomotic high-risk Ivor Lewis esophagectomy patients were treated with preemptive intraluminal Eso-SPONGE treatment for 5 days from the day of surgery, with only one anastomotic leak, which is considerably lower than expected. ${ }^{4}$ With the new insights from Klevebro et al. published in this issue of Annals of Surgical Oncology, we may have sharp enough selection tools to pinpoint patients with a sufficiently high risk of anastomotic complications to justify a nonrandomized phase II trial of preemptive intraluminal vacuum sponge treatment.

\section{REFERENCES}

1. Klevebro F, Elliot JA, Slaman A, Vermeulen BD, Kamiya S, Rosman C, Gisbertz SS, Boshier PR, Reynolds JV, Rouvelas I, Hanna GB, van Berge Henegouwen MI, Markar SR. Cardiorespiratory comorbidity and postoperative complications following esophagectomy: a European multicenter cohort study. Ann Surg Oncol. 2019. https://doi.org/10.1245/s10434-019-07478-6.

2. Reynolds JV, Preston SR, O'Neill B, et al. ICORG 10-14: NEOadjuvant trial in Adenocarcinoma of the Esophagus and oesophagoGastric junction International Study (Neo-AEGIS). BMC Cancer. 2017;17(1):401.

3. Hoeppner J, Lordick F, Brunner T, et al. ESOPEC: prospective randomized controlled multicenter phase III trial comparing perioperative chemotherapy (FLOT protocol) to neoadjuvant chemoradiation (CROSS protocol) in patients with adenocarcinoma of the esophagus (NCT0250986). BMC Cancer. 2016;16:503.

4. Gubler C, Vetter D, Schmidt HM, Müller PC, Morell B, Raptis D, Gutschow CA. Preemptive endoluminal vacuum therapy to reduce anastomotic leakage after esophagectomy: a game-changing approach? Dis Esophagus. 2018. https://doi.org/10.1093/dote/d oy126.

Publisher's Note Springer Nature remains neutral with regard to jurisdictional claims in published maps and institutional affiliations. 